\title{
Oral Health Care Situation of Dependent Older People: Older Health Care Stakeholders' Perspectives
}

\author{
Tipruthai Prayoonwong ${ }^{1,2}$, Tidawan Wiwatkhunupakan ${ }^{1}$, Duangruedee Lasuka ${ }^{3}$ and Patcharawan Srisilapanan ${ }^{1,4^{*}}$ \\ ${ }^{1}$ Family and Community Dentistry Department, Faculty of Dentistry, Chiang Mai University, Chiang Mai, Thailand \\ ${ }^{2}$ Department of Preventive Dentistry, Faculty of Dentistry, Naresuan University, Phitsanulok, Thailand \\ ${ }^{3}$ Department of Medical Nursing, Faculty of Nursing, Chiang Mai University, Chiang Mai, Thailand \\ ${ }^{4}$ Center of Excellent in Dental Public Health, Chiang Mai University, Chiang Mai, Thailand
}

*Corresponding author: Patcharawan Srisilapanan, Center of Excellent in Dental Public Health, Chiang Mai University, Suthep Road, Muang, Chiang Mai, 50200, Thailand, Tel: +66 5394 4487; E-mail: patcharawana@gmail.com

Rec date: Jul 24, 2015; Acc date: Aug 30, 2015; Pub date: Sep 1, 2015

Copyright: (C) 2015 Prayoonwong T, et al. This is an open-access article distributed under the terms of the Creative Commons Attribution License, which permits unrestricted use, distribution, and reproduction in any medium, provided the original author and source are credited.

\begin{abstract}
Objective: To explore the oral health care situation of dependent older people in Tambon Tha Pla Duk, Amphur Mae Tha, Lamphun Province in the north of Thailand from the perspectives of older health care stakeholders.

Material and Method: Data were collected through focus group discussion with key stakeholders in older peoples' health care. Supplementary data were collected through a triangulation of in-depth interviews, selfadministered questionnaires, and participant observations with field notes. Data analysis was carried out by using qualitative approaches. The data from all processes were coded, grouped, interpreted, and thematically analyzed for emerging themes.
\end{abstract}

Results: The oral health care situation of dependent older people identified by the stakeholders were categorized into four issues: 1) Stakeholders' perceptions about the importance of oral care for dependent older people, 2) The current oral health services and access to oral health services for dependent older people, 3) The problems identified by the stakeholders regarding oral care, and 4) The needs of the stakeholders regarding oral care. There were three main issues needing urgent consideration to improve the oral health care of the dependent older people in this sub-district: 1) No specific oral health care and services were available for the dependent older people. 2) There would have to be an improvement of the stakeholders' knowledge, skills, and positive attitudes regarding older peoples' oral care. 3) Increased awareness of older peoples' oral health care in the community was necessary.

Conclusion: The oral health care situation of dependent older people identified by the stakeholders should be considered important in order to develop an effective geriatric oral health program in this sub-district.

Keywords: Oral health care; Oral health services; Dependent older people; Stakeholders

\section{Introduction}

The need to maintain good oral health in Thai older people is gaining more attention than ever as more Thai people live longer. Oral health remains an important issue for older people, because of the continued increase in the number of Thai older people. According to population projections for Thailand from 2010 to 2040, derived from the Annual Report Situation of Thai Older People 2012, the number of Thai older people who aged 60 and over has increased continuously, from 13.2 percent of the total population in 2010, and is likely to escalate to around 32.1 percent of the total population by 2040 [1]. Moreover, the percentage of the oldest-aged population (aged 80 and over) is likely to rise sharply from approximately 12.7 percent in 2010 to 19.1 percent of the total older people population by 2014 [1].

The longer-living older people are likely to have a higher risk of chronic illnesses, infirmities or disabilities. This will cause them to depend more on others in their daily activities. The number of highly dependent older people was 140,000 in 2009 and it is expected to double to 280,000 by 2024 [2]. When older people become more dependent, they invariably have unhealthy mouths and teeth. Visual impairment, loss of manual dexterity, cognitive impairment, and depression are the main causes of poor oral health [3]. Poor oral health in dependent older people has a significant impact on their general health and a negative effect on their quality of life [4]. Oral problems can cause pain, infection, inability to eat or communicate, may increase the risk of complications or lead to life-threatening conditions - including malnutrition, dehydration, brain abscesses, valvular heart disease, joint infections, cardiovascular disease, diabetes mellitus, and aspiration pneumonia [4,5].

In Thailand, most dependent older people receive informal care provided by their families, relatives, volunteers (such as village health volunteers (VHVs)) [6-8], and/or health care professionals from the lowest level of public health services, which are called Health Promoting Hospitals (HPHs) $[8,9]$. However, VHVs as well as health care professionals in HPHs may provide some simple oral health care to dependent older people or caregivers, but they may lack the appropriate knowledge and skills to perform proper oral health care. 
In addition, in order to solve health care problems effectively, the cause of the problems, the way of living, community bonding, and maximizing the community resources and capabilities must be studied first [10]. Therefore, it is important to address community oral health problems and their needs for dependent older peoples' care. These identified problems and needs could be used as the basis for developing effective potential problem solving strategies and/or for developing geriatric oral health program in the community.

Thus, the aim of this study is to explore the older health care stakeholders' perspectives of the oral health care situation for dependent older people in Tambon Tha Pla Duk, Amphur Mae Tha, Lamphun Province, Thailand. This study is part of the main project entitled "The Development of a Community-Based Oral Health Care Model for Thai Dependent Older People".

\section{Materials and Methods}

This study was conducted in Tambon Tha Pla Duk, Amphur Mae Tha, Lamphun Province, Thailand. This sub-district was purposely selected, because it had a high number of effective community projects for older people.

Data were collected through focus group discussion with the key stakeholders in older peoples' health care. Supplementary data were collected through a triangulation of in-depth interviews, selfadministered questionnaires, and participant observations with field notes.

In the first stage of data collection, identification of the older health care stakeholders occurred through in-depth interviews with the director of Tha Pla Duk HPH and two health care professionals in Tha Pla Duk HPH. The stakeholders were selected based on their experience and the amount of responsibility they had for older peoples' care.

In the second stage, self-administered questionnaires and in-depth interviews were conducted with thirty-one stakeholders (three health care professionals in Tha Pla Duk HPH, three VHVs, two dental health professionals (from neighboring HPHs), three leaders of senior citizen clubs, two community leaders, one public health officer, and seventeen dependent older people and/or caregivers) to identify the oral health care situation for dependent older people. Their responses were then collected and collated. Guiding questions for in-depth interviews with dependent older people and/or family caregivers were as follows:

Do you have any oral health problems? If yes, please tell me about your problem(s).

What is the oral health care you need from the HPH?

Guiding questions for self-administered questionnaires with other stakeholders are as follows:

Is oral care important for dependent older people? If yes, please give the reason(s).

What are the oral health services for the dependent older people?

How do the dependent older people access oral health services?

What are the oral health care problems for dependent older people?

What are the needs regarding oral health care for the dependent older people?
What are your suggestions about oral health care for the dependent older people?

The third stage of data collection, participant observations were used to obtain a clearer understanding of the Home Health Care project by observing the Home Health Care team in an older peoples' care home. The researcher observed how the Home Health Care team worked in a genuine situation. Field notes were written up of the activities carried out by the Home Health Care team during the observation periods.

In the final stage of data collection, a focus group discussion was conducted with thirty stakeholders (three health care professionals in Tha Pla Duk HPH, seven VHVs, two dental health professionals (from neighbor HPHs), eight leaders of senior citizen clubs, four community leaders, three local government organizational officers, two caregivers, and one dependent older person). This stage took place to gain a better understanding and confirmation about the current situation of oral health care for dependent older people in this sub-district. The main issues for the focus group discussion were as follows:

Stakeholders' perceptions about the importance of oral care for dependent older people;

The current oral health services and access to oral health services for dependent older people;

The problems identified by the stakeholders regarding oral care;

The needs of the stakeholders regarding oral care.

After the four stages of data collection, data analysis was carried out. Audio-recordings and field notes were transcribed. The data from all processes were coded, grouped, interpreted and thematically analyzed for emerging themes and patterns independently by the researcher (TP).

\section{Results}

In this study, the stakeholders played important roles in oral care for dependent older people. Therefore, the identification of the stakeholder was the first priority.

\section{Identification of the Stakeholders}

The stakeholders were defined as people in the community responsible for all aspects of care for the older people. The important roles of the stakeholders were providing relevant information and suggestions to refine the current oral health care situation of dependent older people.

The stakeholders consisted of 1) dependent older people under the health care system and the responsibility of Tha Pla Duk HPH, 2) caregivers, 3) health care professionals in Tha Pla Duk HPH, 4) VHVs, 5) dental health professionals (from neighboring HPHs), 6) leaders of the senior citizen clubs, 7) community leaders, 8) local government officers, and 9) public health officers.

\section{Main Finding: The Oral Health Care Situation of Dependent Older People}

The current situation of oral health care in this sub-district was as follows: 


\section{The stakeholders' perceptions about the importance of oral care for dependent older people}

Most of the stakeholders agreed that oral health care in dependent older people is important. The reasons were given as follows:

Good oral hygiene could reduce other complications or other symptoms/chronic diseases;

Good oral health leads to good general health;

An oral cavity is a pathway to the body; and

Dependent older people must have special care because of their dependent condition and oral problems can occur if oral hygiene is not maintained (e.g. tooth loss, pain, and problems with eating).

\section{The current oral health services and access to oral health services for dependent older people}

Oral health services for older people in this sub-district were summarized as follows: 1) providing older people oral health examinations by dental health professionals (from neighboring HPHs) or health care professionals in Tha Pla Duk HPH at the same time as annual health check-ups, 2) educating or advising on oral health care to the older people and their caregivers by health care professionals in Tha Pla Duk HPH and VHVs, and 3) undertaking oral health activities with senior citizen' clubs such as providing training in older peoples' oral care.

The specific health care service for dependent older people in this sub-district was the Home Health Care project. For Tha Pla Duk HPH, this project focused on providing health services to older people in their own home to improve their physical and mental health, and optimal social conditions via an outreach team. This team included health care professionals in Tha Pla Duk HPH and community groups (VHVs and older people members in senior citizen' clubs). There were no dental health professionals working in Tha Pla Duk HPH. Oral health care for dependent older people consisted of only simply recommending the use of a mouthwash. There were no specific oral care provisions or services for dependent older people.

\section{The problems identified by the stakeholders regarding oral care}

The problems of oral health care for dependent older people mentioned by the stakeholders were oral health problems; caregivers' stressful conditions; barriers associated with accessibility; lack of dental health professionals; low awareness of the importance of oral health; dental beliefs and attitudes; lack of oral care knowledge and practical skills; and insufficient and inappropriate oral care instruments.

\section{Oral Health Problems}

Oral health problems of dependent older people reported were bad breath; severe tooth mobility; denture problems (e.g. loose-fitting dentures; non-professionally made dentures; and no dentures); dry mouth; swallowing difficulties; choking while swallowing; edentulousness; tooth decay; and gum disease.

\section{Caregivers' stressful conditions}

Almost all caregivers in this sub-district were family members, who invariably worked under stressful conditions. The results indicated that most family caregivers tended to have high workloads in addition to care giving, which involved playing multiple roles. Moreover, the stakeholders reported that the majority of caregivers lacked appropriate knowledge and skills to perform daily oral health care for their dependent older people.

\section{Barriers associated with accessibility}

Issues around access emerged as a barrier for dependent older people in seeking and receiving dental care. The stakeholders indicated that travelling to the dental health professionals represented a significant barrier for dependent older people, using both private and public transport. In the majority of cases, the older people did not own a car or have access to one; therefore, they relied on public transport or on other people to take them to dental clinics. Moreover, a problem was identified relating to a lack of knowledge about where the services were located and how to access them.

\section{Lack of dental health professionals}

There were no dental health professionals working in Tha Pla Duk $\mathrm{HPH}$. It was considered a problem in terms of providing oral care for dependent older people in the community. The stakeholders stated that oral care for older people was a specific task that was required oral care education and oral health services from dental health professionals.

\section{Low awareness of the importance of oral health}

The stakeholders indicated that people in the community focused more on general health than oral health. They demonstrated less awareness of the importance of oral health. Some of them neglected oral care, especially for the dependent older people, because they thought that dependency was an end of life situation; and therefore, the older people with this condition did not need oral care.

\section{Dental beliefs and negative attitudes}

The findings on dental beliefs and negative attitudes were as follows: 1) There was a belief that tooth loss was inevitable or oral problems were part of the aging process. 2) People in the community believed and depended on traditional doctors when they suffered from dental problems. 3) The negative perceptions of dental treatment found were fear of pain related to dental treatment; fear of the human immunodeficiency virus (HIV) infection; and the long length of time involved in receiving dental treatment.

\section{Lack of oral care knowledge and practical skills}

Most stakeholders reported that they did not have enough knowledge about oral health or know how to clean the mouth of a dependent older person properly. They indicated that lack of oral care knowledge and practical skills was a significant problem when providing oral care for dependent older people in the community.

\section{Insufficient and inappropriate oral care instruments}

The final problem identified was insufficient and inappropriate oral care instruments. The stakeholders mentioned that they did not have 
appropriate or adequate oral care instruments necessary to serve the dependent older people - especially bedridden older people.

\section{The Needs of the Stakeholders Regarding Oral Care}

The oral health care needs identified by the stakeholders were: the need for dental treatment; adequate and appropriate oral care instruments and materials; proper oral health education and oral care training of persons related to dependent older people; the creation of a supportive environment with regard to oral care through activities which could be linked to senior citizen' clubs; budget allocation for oral health for poor older people; and effective dental care systems (oral health screening, regular oral health check-ups, follow-ups and evaluations, and consultations).

The stakeholders also suggested that oral health care leaders and expert VHVs in oral health should be created in the community. Moreover, there was also a perceived need for increased awareness about the importance of oral health to other people in the community.

\section{Discussion}

\section{The stakeholders' perceptions about the importance of oral care}

The findings suggest that the stakeholders were aware of the importance of oral care for dependent older people, and they could make connections between oral health and general health. However, the researcher in this study was a dentist; participants may answer directly with the field work of the researcher.

The importance of oral health of older people is often misunderstood and neglected [11], especially in older people with high dependency (e.g. dementia) who cannot easily express their dental pain and problems to others [11]. This situation is in accordance with the finding of this study that oral health care issues for dependent older people were neglected by older people themselves, caregivers, and people in the community. Thus, the stakeholders suggested that it was necessary to advocate the importance of oral health to people in the community and to create a supportive environment about oral care via activities linked with senior citizen clubs.

\section{Current oral health services and access to oral health services for the dependent older people}

When considering all oral health services for the older people in this sub-district, it seemed that some of those services were not suitable for the dependent older people who had limited access to HPH. The service provided, especially to the dependent older people in this subdistrict, was the Home Health Care project. Thus, oral health services for the dependent older people were only home-based care. However, the Home Health Care project in this sub-district mainly focused on providing general health services to older people in their own homes.

The shortage of dental health professionals in the HPH was a major problem to providing oral health prevention for dependent older people in the Home Health Care project. Health care professionals in $\mathrm{HPH}$ or VHVs may provide some oral health care to dependent older people or educate caregivers at home. However, the stakeholders mentioned that they may lack the appropriate knowledge and skills to perform oral health care, especially for the dependent older people. Many researchers recommend that improving oral health care knowledge and an increase in skills of caregivers and other health professionals can improve the oral health status of older people [12-15].

\section{The Problems Identified by the Stakeholders Regarding Oral Care}

\section{Oral health problems}

Older people becoming dependent may face visual impairment, loss of manual dexterity, cognitive impairment, systemic and psychiatric illnesses, and adverse effects from multiple medications $[3,16]$. All of these health conditions are identified as a potential barrier to oral health care. Health status limits a person's ability to perform adequate oral care for maintaining a healthy mouth and limits opportunities to seek out and receive dental care or treatment.

\section{Caregivers' stressful conditions}

Caregivers were forced to deal with a high workload, stress, and lack of oral care knowledge and skills. Some caregivers had to take care of both their children and their parents at the same time. It is not a good situation for dependent older people, especially those in poor families, who face many problems such as lack of knowledge, physical and mental health problems, and a low economic status of the caregiver to cope if the need for older people's care continues for a long time [17]. Therefore, it is necessary to offer formal and other informal (besides "family care") care to assist and support families in addressing the needs of the older people and their families.

\section{Oral health care barriers: dental beliefs and negative attitudes, lack of oral care knowledge and practical skills, and insufficient and inappropriate oral care instruments}

The dental beliefs and negative attitudes identified by the stakeholders in this study were found to be negative perceptions and misunderstandings about oral health care for dependent older people. Lack of knowledge, negative dental attitudes, and low expectations about oral health and its value influence care-seeking behavior and could result in care being deferred or neglected entirely $[4,18]$. Moreover, lack of essential and basic oral care instruments can also create a significant barrier to maintaining good oral health for dependent older people [4].

Most stakeholders reported that they did not have enough knowledge about oral health, or know how to clean the mouth of a dependent older person properly. Improved oral care knowledge, and more positive attitudes towards dependent people, as well as training to ensure adequate skills of carers is likely to improve the oral health and quality of life of dependent older people.

To achieve the oral health care needs of the stakeholders requires new approaches. Successful oral care for dependent older people should consist of the following: an oral health assessment, daily oral care and treatment, educating caregivers, use of varied and individual educational techniques, and evidence-based preventive strategies [19].

\section{The needs of the stakeholders regarding oral care and problem solving strategies}

The responses from the stakeholders regarding oral health problems and their needs were used as basic information to develop problem- 
Citation: Prayoonwong T, Wiwatkhunupakan T, Lasuka D, Srisilapanan P (2015) Oral Health Care Situation of Dependent Older People: Older Health Care Stakeholders' Perspectives . Gerontol Geriatr Res S4: 009. doi:10.4172/2167-7182.S4-009

Page 5 of 5

solving strategies for dependent older people in this study. In striving to improve the oral health care system and services, it is important to build upon what is already there in the local communities, and to create a sense of ownership of the activities and results. All older health care stakeholders need to be fully aware of oral health for dependent older people. They also need to be involved in the efforts to strengthen the oral health care system and services including the dependent older people, caregivers, health care professionals in HPH, VHVs, dental health professionals, leaders of senior citizen clubs, community leaders, local government officers, and public health officers. In this way, the oral health care system and services can evolve in a way that is appropriate for the local setting and resources, and can fit in smoothly with local health policies, which may differ from province to province.

\section{Conclusions}

Improved oral health should lead to an improved quality of life. From the findings of this study, there were three main issues needing urgent consideration to improve the oral health care system and services for the dependent older people: 1) No specific oral health care or services were available for the dependent older people. 2) There would have to be an improvement of the stakeholders' knowledge, skills, and positive attitudes regarding older peoples' oral care. 3) Increased awareness of older peoples' oral health care in the community was necessary. Moreover, problem solving strategies identified by the stakeholders should be considered as important information to develop an oral health care program for dependent older people in order to create a sense of ownership of the activities and the leading to an improved situation regarding oral health for dependent older people in this community.

\section{Acknowledgements}

This study was funded by the Faculty of Dentistry and Chiang Mai University. The authors would like to thank all of the participants and staff in Tambon Tha Pla Duk, Amphur Mae Tha, Lamphun for their cooperation.

\section{References}

1. Foundation of Thai Gerontology Research and Development Institute (2012) Annual Report Situation of the Thai Elderly. SS Plus Media Company Limited, Nonthaburi.

2. Srithamrongsawat S, Bundhamcharoen K (2010) Synthesis of long-term care system for the elderly in Thailand [In Thai]. TQP Printing Co., Bangkok.
3. Chalmers JM, Carter KD, Spencer AJ (2003) Oral diseases and conditions in community-living older adults with and without dementia. Spec Care Dentist 23: 7-17.

4. Helgeson MJ, Smith BJ, Johnsen M, Ebert C (2002) Dental considerations for the frail elderly. Spec Care Dentist 22: 40s-55s.

5. Meurman JH, Hamalainen P (2006) Oral health and morbidity-implications of oral infections on the elderly. Gerodontology 23: 3-16.

6. Kespichayawattana J, Jitapunkul S (2009) Health and health care system for older persons. Ageing Int 33: 28-49.

7. Porapakkham Y, Atibodhi S (2002) The synthesis of the national policy on ageing. Bangkok.

8. Yodpet S (2009) The integrations of long term care system for the elderly in Thailand [In Thai]. J Print 2, Bangkok.

9. Auamkul N, Kanshana S, Phirangapaura A (1999) Fact sheet: development of health promoting hospitals in Thailand. Nonthaburi.

10. Nuntaboot K (2006) Nurses of the community, by the community, and for the community in Thailand. Regional Health Forum 10: 11-28.

11. Lin CY, Jones DB, Godwin K, Godwin RK, Knebl JA, et al. (1999) Oral health assessment by nursing staff of Alzheimer's patients in a long-termcare facility. Spec Care Dentist 19: 64-71.

12. Petersen PE, Yamamoto $\mathrm{T}$ (2005) Improving the oral health of older people: the approach of the WHO global oral health programme. Community Dent Oral Epidemiol 33: 81-92.

13. Frenkel H, Harvey I, Needs K (2002) Oral health care education and its effect on caregivers' knowledge and attitudes: a randomised controlled trial. Community Dent Oral Epidemiol 30: 91-100.

14. Frenkel H, Harvey I, Newcombe RG (2001) Improving oral health in institutionalised elderly people by educating caregivers: a randomised controlled trial. Community Dent Oral Epidemiol 29: 289-97.

15. Van Der Putten GJ, De Visschere L, Schols J, de Baat C, Vanobbergen J (2010) Supervised versus non-supervised implementation of an oral health care guideline in (residential) care homes: a cluster randomized controlled clinical trial. BMC Oral Health 10: 17.

16. Schwartz M (2000) The oral health of the long-term care patient. Ann Long-Term Care 8: 41-46.

17. The National Health Commission of Thailand (2009) The 2nd National Health Assembly (agenda 3.3): development of long-term care for dependent elderly people. Nonthaburi.

18. Cohen-Mansfield J, Lipson S (2002) The underdetection of pain of dental etiology in persons with dementia. Am J Alzheimers Dis Other Demen 17: 249-53.

19. Frenkel H, Matthews DC, Nitschke I (2011) Prevention of oral diseases for a dependent population., in Oral healthcare and the frail elder: a clinical perspective. 